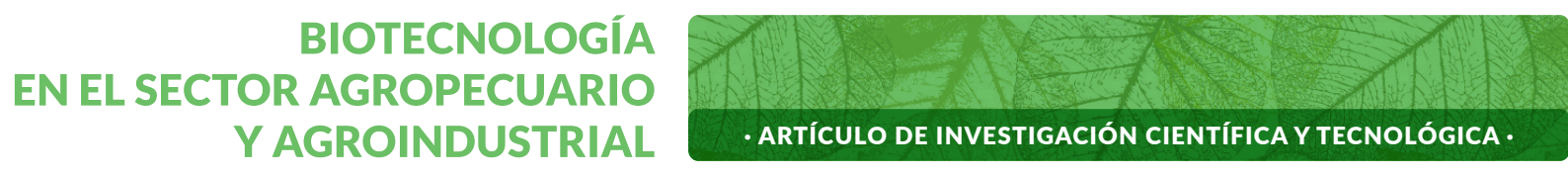

Vol. 16 No 2 • Julio - Diciembre 2018 • ISSN - 1692-3561 · ISSN-e 1909-9959 · doi: http://dx.doi.org/10.18684/bsaa.v16n2.100

\title{
Estudios de estabilidad térmica sobre el ingrediente farmacéutico activo de la vacuna gavac ${ }^{\circledR}$
}

\section{Thermal stability studies over active pharmaceutical ingredient of gavac ${ }^{\circledR}$ vaccine}

\section{Estudos de estabilidade térmica de ingrediente farmacêutico ativa da vacina gavac ${ }^{\circledR}$}

\author{
RUTDALI M. SEGURA-SILVA ${ }^{1}$, ARLENIS ALFARO-MARTÍNEZ², \\ DIASMARYS SALINAS-RODRIGUEZ³ , ALAIN MOREIRA-RUBIO4 ${ }^{4}$ AMAURY PÉREZ-SÁNCHEZ
}

\section{RESUMEN}

En la actualidad los estudios de estabilidad térmica constituyen etapas fundamentales dentro del desarrollo de procesos biotecnológicos, ya que mediante ellos se determinan las condiciones óptimas a tomar en cuenta durante el manejo y almacenamiento de las diferentes vacunas obtenidas. En el presente trabajo se llevan a cabo 2 estudios de estabilidad térmica sobre el Ingrediente Farmacéutico Activo

Recibido para evaluación: 7 de Noviembre de 2017.

Aprobado para publicación: 31 de Abril de 2018.

1 Centro de Ingeniería Genética y Biotecnología de Camagüey, Departamento de Producción. Magíster en Análisis de Procesos Químicos. Camagüey, Cuba.

2 Centro de Ingeniería Genética y Biotecnología de Camagüey, Departamento de Mantenimiento. Ingeniero Químico. Camagüey, Cuba.

3 Centro de Ingeniería Genética y Biotecnología de Camagüey, Departamento de Control de Calidad. Licenciada en Química. Camagüey, Cuba.

4 Centro de Ingeniería Genética y Biotecnología de Camagüey, Departamento de Control de Calidad. Magíster en Medio Ambiente. Camagüey, Cuba.

5 Universidad de Camagüey, Facultad de Ciencias Aplicadas, Departamento de Ingeniería Química. Ingeniero Químico. Camagüey, Cuba.

Correspondencia: amauryps@nauta.cu 
(IFA) de la vacuna Gavac $^{\circledR}$, relacionados con: 1) Incremento del tiempo de vigencia del IFA hasta un período de 30 días a una temperatura entre 2 y $8^{\circ} \mathrm{C}$, y 2) Establecimiento el número de veces que el IFA se puede congelar y descongelar por un período de 90 días a una temperatura de $-20^{\circ} \mathrm{C}$, todo ello sin afectar los parámetros de calidad establecidos por el sistema de Control de Calidad del Centro de Ingeniería Genética y Biotecnología (CIGB) de Camagüey. De acuerdo con los resultados obtenidos se puede concluir que el IFA de la vacuna $\operatorname{Gavac}^{\circledR}$ es estable por un período de 30 días a una temperatura de $2-8^{\circ} \mathrm{C}$, y que puede ser sometido a 5 congelaciones y descongelaciones por un período de tiempo de 90 días $a-20^{\circ} \mathrm{C}$ sin que se afecten negativamente sus principales parámetros de calidad.

\section{ABSTRACT}

Currently the thermal stability studies constitute fundamental stages during the development of biotechnological processes, since through them the optimum conditions that must be taken into account during the storage and handling of the different vaccines obtained are determined. In the present work two thermal stability studies are carried out on the Active Pharmaceutical Ingredient (API) of Gavac ${ }^{\circledR}$ vaccine, related with: 1) Increment of the validity time of the API to 30 days at a storage temperature of $2-8^{\circ} \mathrm{C}$, and 2) Implementation of the number of times that the API can be frozen/defrosted over a time period of 90 days at a temperature of $-20^{\circ} \mathrm{C}$, without affecting the quality parameters established by the Quality Control system of the Center of Genetic Engineering and Biotechnology of Camagüey. According to the results obtained it can be concluded that the API of Gavac ${ }^{\circledR}$ vaccine is stable for 30 days at a temperature of $2-8^{\circ} \mathrm{C}$, and that it can be subjected to 5 freezing/defrosting operations for a time period of 90 days at $-20^{\circ} \mathrm{C}$ of temperature without affecting negatively its main quality parameters.

\section{RESUMO}

Hoje estudos de estabilidade térmica são elementos fundamentais para o desenvolvimento de processos biotecnológicos, porque através deles as condições ideais estão determinadas a ter em conta durante o manuseio e armazenamento de diferentes vacinas obtidas. Neste trabalho realizado dois estudos de estabilidade térmica do ingrediente farmactico activo (IFA) da vacina Gavac $^{\circledR}$ relacionada com: 1) aumentar a vida útil da IFA até um período de 30 dias a uma temperatura de $2-8^{\circ} \mathrm{C}$, e 2) definir o número de vezes que o IFA pode congelar e descongelar durante um período de 90 dias a uma temperatura de $-20^{\circ} \mathrm{C}$, sem afectar todos os parâmetros de qualidade estabelecidos pelo sistema de controle de qualidade de Centro de Engenharia Genética e Biotecnologia (CIGB), em Camagüey. De acordo com os resultados pode concluirse que a IFA de vacina $\mathrm{Gavac}^{\circledR}$ es estável durante um período de 30 dias a $2-8^{\circ} \mathrm{C}$, e podem ser sujeitas a 5 congelamento $e$ descongelamento durante um período de tempo 90 dias $-20^{\circ} \mathrm{C}$, sem afetar negativamente seus principais parâmetros de qualidade.

\section{PALABRAS CLAVES:}

Estabilidad térmica, Ingrediente Farmacéutico Activo, Control de calidad.

\section{KEYWORDS:}

Thermal stability, Active Pharmaceutical Ingredient, Quality control.

\section{PALAVRAS-CHAVE:}

Estabilidade térmica, Ingrediente Farmacêutico Activo, Qualidade. 


\section{INTRODUCCIÓN}

Las vacunas constituyen productos biológicos complejos que pueden degradarse debido a su almacenamiento bajo condiciones inadecuadas de temperatura, lo cual se intensifica a menudo con la aplicación de altas temperaturas. Un número elevado de productos farmacéuticos y biológicos utilizados a nivel mundial tales como insulina, vacunas, agentes quimioterapéuticos, antibióticos - son sensibles a la temperatura [1].

Consecuentemente, estos requieren condiciones especiales de producción, almacenamiento, y transporte para evitar su exposición a factores medioambientales inadecuados, con el fin de garantizar el mantenimiento de su estabilidad en el tiempo [2]. De esta manera, el establecimiento de los parámetros de estabilidad térmica de estos productos durante su almacenamiento constituye un factor de gran importancia para asegurar su eficacia a lo largo del tiempo de vigencia establecido. El objetivo de un estudio de estabilidad radica en suministrar evidencias acerca de cómo varían ciertos parámetros de calidad de un Ingrediente Farmacéutico Activo (IFA) o de un Producto Farmacéutico Terminado (PFT) en el tiempo, bajo la influencia de determinados factores medioambientales tales como temperatura, humedad y luz. El programa de estabilidad de una vacuna también puede incluir la evaluación de la interacción existente entre el IFA y determinados factores tales como los excipientes y adyuvantes usados, los sistemas de sellado y cierre de los envases, así como también los materiales de empaque [2]. También se puede evaluar la interacción entre sí de dos o más IFA contenidas en un mismo PFT. Como resultado del estudio de estabilidad térmica se conoce la durabilidad en el tiempo del PFT, así como también las condiciones de almacenamiento más adecuadas [3].

En años recientes los estudios de estabilidad han estado relacionados con el desarrollo de métodos para extender la estabilidad de antígenos en vacunas. Esto resulta específicamente importante en países en vías de desarrollo en los cuales el acceso a equipos y medios de refrigeración adecuados no siempre resulta factible. En la actualidad, tanto el personal médico como los investigadores y científicos se encuentran desarrollando nuevas rutas o vías para prolongar la estabilidad térmica y el tiempo de vida de las vacunas, usualmente mediante la adición de aditivos y compuestos adyuvantes, entre los cuales se pueden mencionar nanopartículas de oro aniónicas y polietilénglicol en vacunas conteniendo adenovirus tipo 5 [4], o excipientes tales como L-leucina, lactosa-trehalosa y manitol/dextrana en vacunas conteniendo el vector adenoviral humano recombinante tipo 5 [5].

La estabilidad térmica de vacunas ha sido evaluada por diferentes autores. Por ejemplo, se evaluó la termoestabilidad de la vacuna contra la fiebre amarilla, tanto en su forma liofilizada como después de la reconstitución, exponiéndolas a temperaturas en el rango de 8 a $45^{\circ} \mathrm{C}$ [6]. Por otro lado, emplearon el ensayo de hemoaglutinación para determinar la estabilidad térmica a $36^{\circ} \mathrm{C}$ de las cepas Hitchner-B1 (B1), LaSota (L) y Komarov (K) de la vacuna contra la enfermedad de Newcastle [7]. También estudiaron la influencia de la humedad residual sobre la estabilidad térmica de 61 lotes de la vacuna contra el Sarampión, las Paperas y Rubeola, empleando un método acelerado consistente en mantener las vacunas a $37^{\circ} \mathrm{C}$ de temperatura por 7 días [8]. En otro estudio se evaluó la termoestabilidad del virus de la enfermedad de Newcastle, a partir de la aplicación de tres ciclos de temperatura: 1) $25^{\circ} \mathrm{C}$ por 36 días; 2) $37^{\circ} \mathrm{C}$ por 29 días, y 3) $56^{\circ} \mathrm{C}$ por un período de tiempo oscilando entre 5 minutos y 8 horas [9]. Por último, efectuaron estudios de estabilidad térmica acelerada $\left(30^{\circ} \mathrm{C}\right.$ por 7 días) y en tiempo real $\left(-20^{\circ} \mathrm{C}\right.$ por 24 meses) sobre un candidato vacunal contra la malaria, obteniendo como resultado que la misma era termoestable para ambos estudios efectuados [10].

En la actualidad el Centro de Ingeniería Genética y Biotecnología (CIGB) de Camagüey produce el inmunógeno Gavac $^{\circledR}$ empleada para erradicar las especies de garrapata (Boophilus microplus) presentes en el ganado vacuno. A menudo se presentan contingencias durante la ejecución del plan de operaciones, lo cual traía consigo paradas productivas que afectaban el cumplimiento del cronograma de producción del inmunógeno Gavac $^{\circledR}$, derivándose de ello el incumplimiento del tiempo de vigencia del IFA Gavac ${ }^{\circledR}$, tanto en el número de descongelaciones, como en el tiempo de conservación a $4^{\circ} \mathrm{C}$ ( 15 días), incurriéndose en no conformidades y por consiguiente en reensayos de los lotes de IFA. Debido a esto se desea incrementar el tiempo de vigencia del IFA de esta vacuna desde los 15 días actuales hasta 30 días a una temperatura de almacenamiento de $2-8^{\circ} \mathrm{C}$, mientras que también se desea conocer el número de veces que el IFA puede ser congelado y descongelado por 
un período de tiempo de 90 días a una temperatura de almacenamiento de $-20^{\circ} \mathrm{C}$. De esta forma, el presente trabajo tiene como objetivo principal mostrar los resultados obtenidos con relación a dos estudios de estabilidad térmica efectuados sobre el IFA del inmunógeno $\mathrm{Gavac}^{\circledR}$, consistentes en: 1) Incrementar el tiempo de vigencia del IFA hasta un período de 30 días a una temperatura de $2-8^{\circ} \mathrm{C}$, y 2) Establecer el número de veces que el IFA puede ser congelado y descongelado sin afectar negativamente los parámetros de calidad establecidos por el sistema de Control de Calidad, para un periodo de tiempo de 90 días y a una temperatura de $-20^{\circ} \mathrm{C}$.

\section{MÉTODO}

\section{Estudio de estabilidad No. 1: Incremento del tiempo de vigencia del IFA}

Para llevar a cabo el estudio de estabilidad número 1 (EE1) se emplearon 3 lotes de IFA (14.1505-2, $14.1505-3$ y $14.1505-4$ ), a los cuales se les tomaron 9 muestras por lote de $10 \mathrm{~mL}$ cada una, durante la etapa de Filtración Estéril del proceso productivo. Las muestras fueron vertidas en tubos roscados de polipropileno de $15 \mathrm{~mL}$ de capacidad, y colocadas a continuación en una cámara frigorífica a $-20^{\circ} \mathrm{C}$ de temperatura por 30 días. En el momento de comenzar el estudio, las muestras fueron colocadas en otra cámara frigorífica a una temperatura de $2-8^{\circ} \mathrm{C}$ y, una vez ubicadas allí, se procedió a tomar muestras a los 15,20 y 30 días. A las muestras tomadas en estos días se le realizaron los ensayos físico-químicos establecidos en la Especificación de Calidad con Número de Parte (NP) 4046 [11], tomando como tiempo cero los ensayos de calidad realizados por el Departamento de Control de Calidad durante el proceso de liberación de los 3 lotes seleccionados.

\section{Estudio de estabilidad No. 2: Número de veces que el IFA puede ser congelado y descongelado}

Los lotes de IFA $14.1505-5 ; 14.1505-6$ y $14.1505-1$ fueron los seleccionados para efectuar el estudio de estabilidad número 2 (EE2). En este caso, y de forma semejante al EE1, las muestras fueron colectadas durante la etapa de Filtración Estéril, tomándose 9 muestras de $150 \mathrm{~mL}$ por lote, las cuales fueron verti- das en tubos roscados de $300 \mathrm{~mL}$ de capacidad. Una vez tomadas, las muestras fueron ubicadas en una cámara frigorífica a $-20^{\circ} \mathrm{C}$ de temperatura por un tiempo de 90 días. Cumplido este tiempo, las muestras presentes en esta cámara frigorífica fueron colocadas en otra cámara frigorífica a una temperatura de 2 $8^{\circ} \mathrm{C} 24$ horas antes de comenzar el EE2, para proceder a su descongelamiento. Una vez descongeladas, las muestras fueron homogenizadas con movimientos suaves del tubo por parte del analista, antes de proceder a la toma de muestra para la realización de los ensayos según la Especificación de Calidad NP 4046. EI EE2 fue efectuado por un período de tiempo de 90 días, llevándose a cabo 5 congelaciones/descongelaciones en total durante ese lapso de tiempo, y tomando también como tiempo cero los ensayos realizados por el Departamento de Control de Calidad durante el proceso de liberación de los 3 lotes seleccionados.

\section{Parámetros a controlar durante los estudios de estabilidad}

A cada una de las muestras tomadas en los dos estudios de estabilidad efectuados se le realizaron los siguientes ensayos físico-químicos de acuerdo con la especificación NP 4046:

- Concentración de proteínas totales

- Pureza

- $\mathrm{pH}$

- Concentración de timerosal

- Características organolépticas y de esterilidad

\section{Determinación de la concentración de proteínas totales}

La concentración de proteínas totales fue determinada por el método de Lowry, según se describe en el Procedimiento Patrón de Operación (PPO) 4.09.002.91 [12].

\section{Determinación de la pureza}

La pureza de las muestras tomadas fue determinada por densitometría SDS-Page, según se describe en el PPO 4.09.004.91 [13] y el PPO 4.09.005.91 [14]. 


\section{Determinación del pH}

Se toman 3 muestras de $10 \mathrm{~mL}$ cada una y se les mide el valor de $\mathrm{pH}$ en un medidor digital de $\mathrm{pH}$ (Mettler Toledo ${ }^{\circledR}$, modelo Seven Compact PH/ION S220). El resultado final se obtiene calculando el promedio de los tres valores de $\mathrm{pH}$ determinados [15].

\section{Determinación de la concentración de timerosal}

La concentración de timerosal fue determinada mediante el PPO 2.31.226.11 [16].

\section{Características organolépticas y esterilidad}

Se determinan evaluando el color y el aspecto cualitativo del Ingrediente Farmacéutico Activo del inmunógeno, el cual debe comportarse como un líquido transparente de color amarillo, sin partículas o microorganismos en suspensión que denoten crecimiento microbiano.

\section{RESULTADOS}

\section{Resultados del Estudio de Estabilidad No. 1}

Concentración de Proteínas Totales. En la figura 1 se muestran los resultados obtenidos durante la determinación de la Concentración de Proteínas Totales (CPT) para los lotes empleados, así como también el criterio de aceptación aprobado para este parámetro según norma NP 4046. Según se puede observar en esta figura, desde el tiempo cero y hasta los 30 días, la CPT se mantuvo siempre por encima del valor mínimo establecido según norma (1 mg/ $\mathrm{mL}$ ), lo cual es un indicativo de que la vacuna puede permanecer por 30 días a una temperatura entre $2-8^{\circ} \mathrm{C}$ sin que se afecte negativamente la CPT.

A los valores de CPT obtenidos para cada lote en los diferentes tiempos del estudio, se les determinó la Desviación Estándar Promedio, obteniéndose de esta manera la dispersión de los valo- res determinados de este parámetro con respecto a la media. Como se puede observar en el cuadro 1 , la dispersión de los valores obtenidos es muy pequeña, por lo que se puede decir que hubo consistencia en los valores de CPT obtenidos a lo largo del estudio de estabilidad efectuado.

Pureza. En la Figura 2 se muestran los resultados obtenidos por SDS-PAGE para el parámetro Pureza, los cuales demuestran que todos los lotes evaluados cumplen con el requisito de calidad especificado según Especificación de Calidad NP 4046 para este parámetro, es decir, banda de $89 \mathrm{kDa} \geq 85 \%$ de pureza. Se comprueba que durante todo el

Cuadro 1. Resultados del Promedio y la Desviación Estándar Media de los valores obtenidos de CPT para los lotes evaluados en el EE1.

\begin{tabular}{|c|c|c|}
\hline Lote & $\begin{array}{c}\text { PROMEDIO } \\
(\mathrm{mg} / \mathrm{mL})\end{array}$ & DESVEST.M \\
\hline $14.1505-2$ & 3,433 & 0,06761841 \\
\hline $14.1505-3$ & 2,666 & 0,04261455 \\
\hline $14.1505-4$ & 4,183 & 0,06344026 \\
\hline
\end{tabular}

Figura 1. Resultados de la concentración de proteínas totales para los lotes evaluados durante el EE1.

\section{Concentración de proteinas totales de los lotes evaluados durante el EE1}

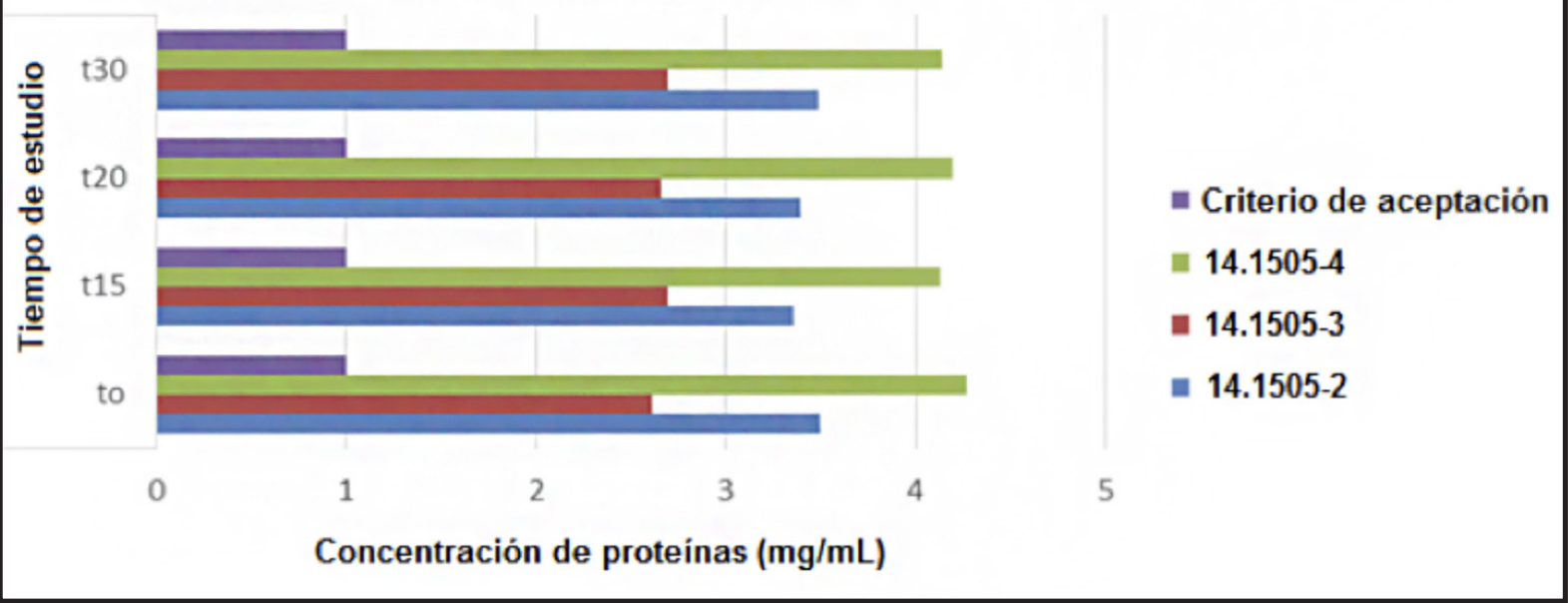


Figura 2. Resultados de la pureza para los lotes evaluados en el EE1.

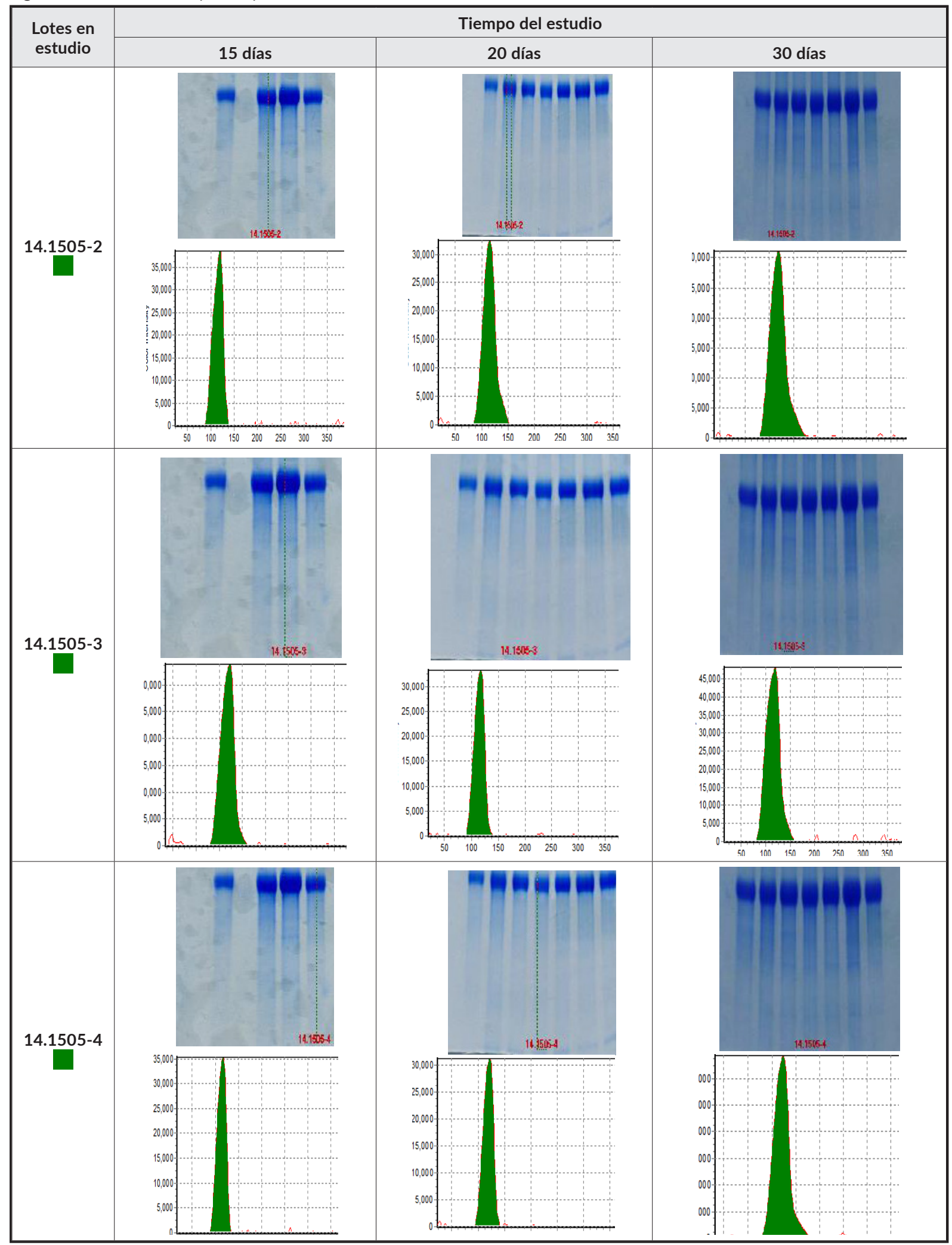


estudio de estabilidad, se mantuvo la presencia de la banda de la proteína $\mathrm{Bm86}$, mientras que la pureza se mantuvo superior al $85 \%$. Lo anterior indica que la pureza del IFA no se afecta al ser almacenada esta por espacio de 30 días a una temperatura entre 2 y $8^{\circ} \mathrm{C}$.

pH y concentración de timerosal. Según se puede observar en las Figura 3 y 4 , tanto el pH como la concentración de timerosal se mantuvieron dentro del rango establecido de acuerdo con la especificación de calidad vigente (NP 4046), la cual es 7,80 - 8,50 para el $\mathrm{pH}$ y menor que $0,05 \mathrm{mg} / \mathrm{mL}$ para la concentración de timerosal. De acuerdo con estos resultados, se puede concluir que el almacenamiento del IFA entre 2 y $8^{\circ} \mathrm{C}$ por 30 días no afecta negativamente tanto el pH como la concentración de timerosal.

Características organolépticas y esterilidad. Durante todo el EE1, las muestras tomadas presentaron las características organolépticas y de esterilidad exigi-

Figura 3. Resultados pH para los lotes evaluados durante el EE1.

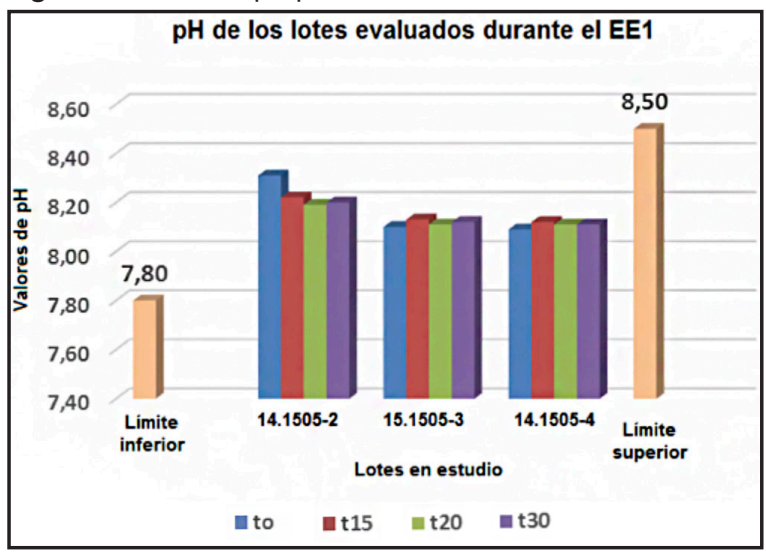

Figura 4. Influencia del tiempo sobre la concentración de timerosal para los lotes evaluados durante el EE1.

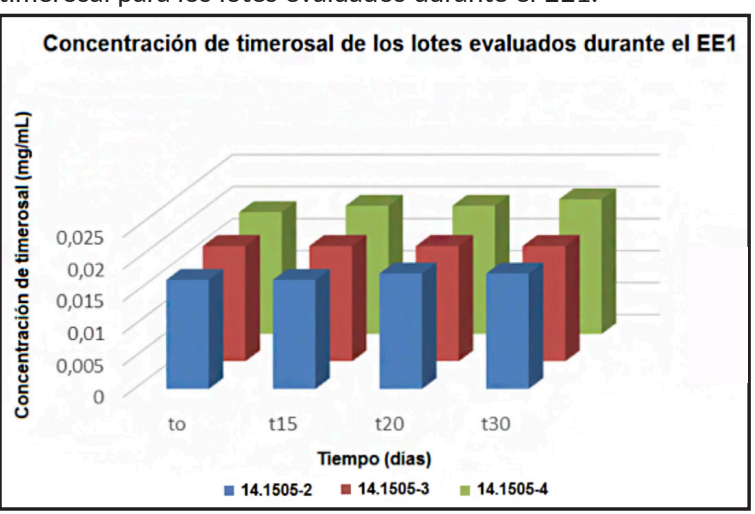

das por la norma NP 4046, es decir, mostraron un aspecto de líquido transparente de color amarillo homogéneo sin partículas en suspensión que denoten crecimiento microbiano, por lo que se puede decir que el almacenamiento entre 2 y $8^{\circ} \mathrm{C}$ por 30 días del IFA de la vacuna Gavac no afectarán tanto sus características organolépticas como de esterilidad.

\section{Resultados del Estudio de Estabilidad No. 2}

Concentración de Proteínas Totales. En la figura 5 se observan los resultados obtenidos de Concentración de Proteínas Totales (CPT) para los lotes seleccionados en este estudio, así como también el criterio de aceptación aprobado de acuerdo con la especificación NP 4046. Según se puede apreciar, las 5 congelaciones /descongelaciones (C/D) efectuadas no afectaron la CPT, obteniéndose siempre valores de CPT por encima del límite mínimo exigido ( $1 \mathrm{mg} / \mathrm{mL}$ ). Cuadro 2

A los valores de CPT obtenidos para cada lote en los diferentes puntos de C/D realizados se les calculó la Desviación Estándar Promedio, a partir de la cual se obtuvo la dispersión de los valores obtenidos con respecto a la media. Como se puede observar en el cuadro 1 , la dispersión resultante es muy pequeña, por lo que se puede decir que hubo consistencia en los valores de CPT obtenidos a lo largo del EE2.

Comportamiento de la pureza. Los resultados de control por SDS-PAGE obtenidos muestran que todos los lotes cumplen con el requisito de calidad especificado en el NP 4046, es decir banda de 89 $\mathrm{kDa} \geq 85 \%$ de pureza, lo cual puede corroborarse a través de la Figura 6. En la misma se puede observar la elevada pureza del IFA obtenido en los lotes en estudio, donde el porciento de pureza estuvo en todos los casos por encima del $95 \%$. Todo esto indica que no hay afectación de la pureza del IFA al efectuarle 5 congelaciones/ descongelaciones a una temperatura de $-20^{\circ} \mathrm{C}$ por un período de tiempo de 90 días.

Cuadro 2. Resultados del Promedio y la Desviación Estándar Media de los valores obtenidos de CPT para los lotes evaluados en el EE2.

\begin{tabular}{|c|c|c|}
\hline Lote & PROMEDIO $(\mathrm{mg} / \mathrm{mL})$ & DESVEST.M \\
\hline $14.1505-5$ & 3,494 & 0,041643730 \\
\hline $14.1505-6$ & 3,109 & 0,081769187 \\
\hline $14.1506-1$ & 3,496 & 0,032634338 \\
\hline
\end{tabular}


Figura 5. Efecto del tiempo sobre la concentración de proteínas para los lotes evaluados en el EE2.

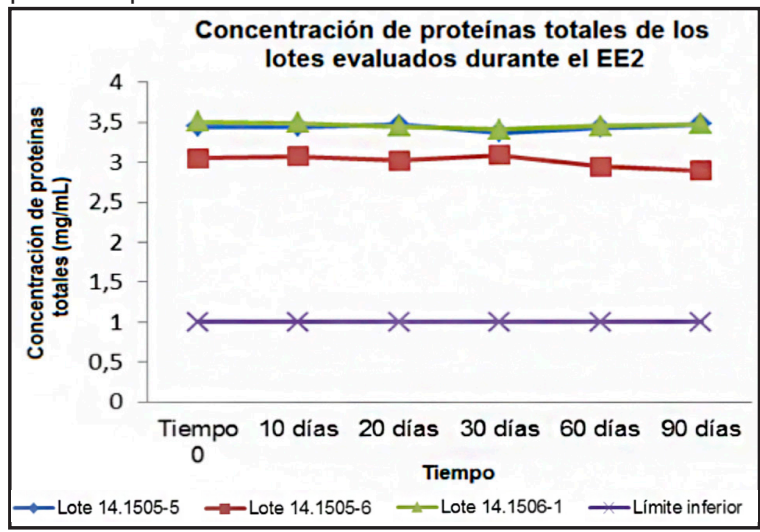

Figura 6. Efecto del tiempo sobre el \% de pureza para los lotes evaluados en el EE2.

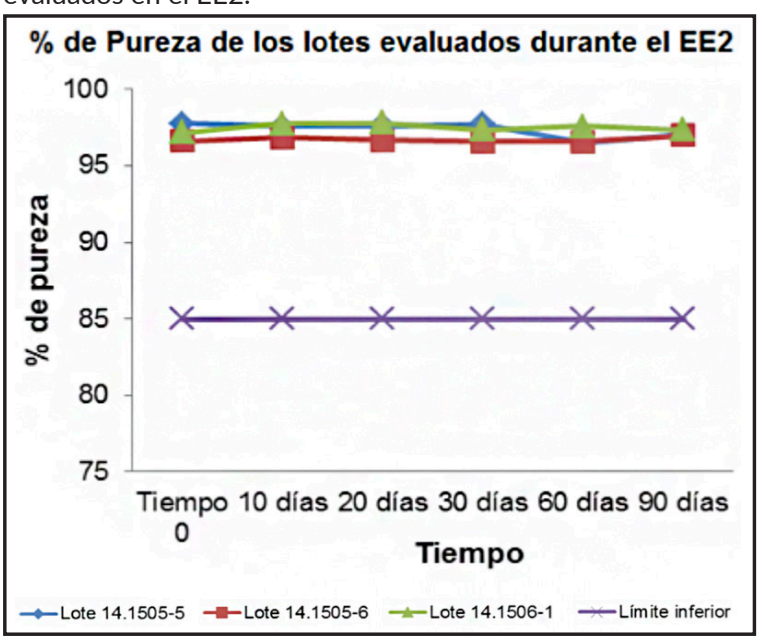

Figura 7. Influencia del tiempo sobre el valor de $\mathrm{pH}$ para los lotes evaluados en el EE2.

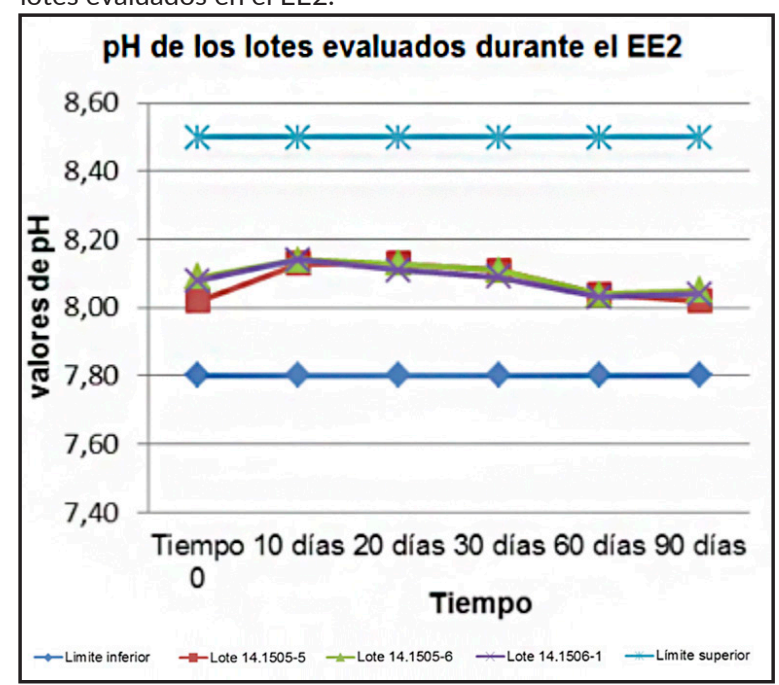

Figura 8. Influencia del tiempo sobre la concentración de timerosal para los lotes evaluados en el EE2.

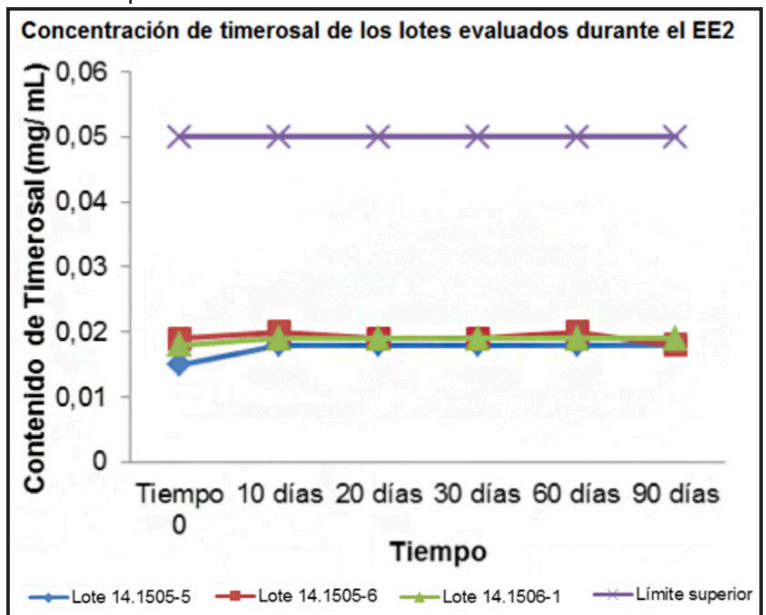

pH y concentración de timerosal. De acuerdo con lo mostrado en las figuras 7 y 8 , tanto el $\mathrm{pH}$ como la concentración de timerosal estuvieron dentro del intervalo establecido por la norma NP 4046 (rango de 7,80 - 8,50 para el $\mathrm{pH}$ y menor de $0,05 \mathrm{mg} / \mathrm{mL}$ para la concentración de timerosal). De esta manera se puede establecer como conclusión que la estabilidad del IFA en cuanto a pH y concentración de timerosal no se afecta al realizarle 5 procedimientos de congelación/descongelación durante 90 días de almacenamiento a $-20^{\circ} \mathrm{C}$ de temperatura.

Características organolépticas y esterilidad. Durante todo el EE2, las características organolépticas y de esterilidad exigidas por la especificación NP 4046 fueron cumplidas, comportándose siempre las muestras tomadas como un líquido transparente de color amarillo homogéneo, sin partículas en suspensión ni indicios de crecimiento microbiano. De esta manera se puede concluir que la realización de 5 congelaciones/descongelación del IFA por un tiempo de 90 días no afecta las características organolépticas y de esterilidad exigidas por las normas de calidad para un producto de este tipo.

Los resultados mostrados en este trabajo concuerdan con los reportados por Canales y colaboradores [17] con respecto a la estabilidad térmica de la vacuna Gavac $^{\circledR}$. 


\section{CONCLUSIONES}

El Ingrediente Farmacéutico Activo de la vacuna Ga$\operatorname{vac}^{\circledR}$ es estable por un período de tiempo de 30 días a una temperatura de 2 a $8^{\circ} \mathrm{C}$. El Ingrediente Farmacéutico Activo de la vacuna Gavac $^{\circledR}$ es estable por un período de 90 días a $-20^{\circ} \mathrm{C}$ sometido a 5 congelaciones y descongelaciones.

\section{REFERENCIAS}

[1] GALAZCA, A., MILSTIEN, J. and ZAFFRAN, $M$. Thermostability of vaccines. Geneva (Italy): World Health Organization, 1998.

[2] WORLD HEALTH ORGANIZATION (WHO). Stability testing of active pharmaceutical ingredients and finished pharmaceutical products. Ginebra (Switzerland): WHO Report No: 953, World Health Organization, 2009.

[3] CHEN, D. and KRISTENSEN, D. Opportunities and challenges of developing thermostable vaccines. Expert Review of Vaccines, 8(5), 2009, p. 547-557.

[4] PELLICCIA, M., ANDREOZZI, P., PAULOSE, J., CAGNO, V. and DONALISIO, M. Additives for vaccine storage to improve thermal stability of adenoviruses from hours to months. Nature Communications, 7(13520), 2016, p. 1-7.

[5] LECLAIR, D.A., CRANSTON, E.D., XING, Z. and THOMPSON, M.R. Evaluation of excipients for enhanced thermal stabilization of a human type 5 adenoviral vector through spray drying. International Journal of Pharmaceutics, 506, 2016, p. 289-301.

[6] ISHAK, R. and HOWARD, C.R. The thermal stability of yellow fever vaccines. Memórias do Instituto Oswaldo Cruz, 85(3), 1990, p. 339-345.

[7] NSSIEN, M.A.S., and ADENE, D.F. Thermostability of Reconstituted Newcastle Disease Virus Strains at $36^{\circ} \mathrm{C}$ Temperature. African Journal of Biomedical Research, 5, 2002, p. 87-89.

[8] SHAHKARAMI, M.K., TAQAVIAN, M., SHAFYI, A., ALIREZAIE, B., ESNA-ASHARI, F., SOLEIMANI, S. and SHAHBAZI, R. Investigation of the Relationship between the Residual Moisture and Thermal Stability of Lyophilized MMR Vaccine. Iranian Journal of Virology, 3(1), 2009, p. 25-28.

[9] CHAKRABORTY, D., GUHA, C., BISWAS, U., CHATTERJEE, A., JANA, P.S., BISWAS, S. and NAHA, B.C. Studies on Thermo stability of
Newcastle Disease Virus (Local Isolate) for Preparation of Vaccine. Journal of Veterinary Science \& Technology, 5(2), 2014, p. 1-12.

[10] FABER, B.W., HELLWIG, S., HOUARD, S., HAVELANGE, N., DROSSARD, J., MERTENS, H. and REMARQUE, E.J. Production, Quality Control, Stability and Pharmacotoxicity of a Malaria Vaccine Comprising Three Highly Similar PfAMA1 Protein Molecules to Overcome Antigenic Variation. PLoS ONE, 11(10), 2016, p. 1-32.

[11] CUBA. CENTRO DE INGENIERÍA GENÉTICA Y BIOTECNOLOGÍA DE CAMAGÜEY (CIGB). Ingrediente Farmacéutico Activo (Bm86) de Gavac $^{\circledR}$. NP 4046. Camagüey (Cuba): Centro de Ingeniería Genética y Biotecnología, 2016.

[12] CUBA. CENTRO DE INGENIERÍA GENÉTICAYBIOTECNOLOGÍA DE CAMAGÜEY (CIGB). Determinación de la concentración de proteínas. Método de Lowry. PPO 4.09.002.91. Camagüey (Cuba): Centro de Ingeniería Genética y Biotecnología, 1991.

[13] CUBA. CENTRO DE INGENIERÍA GENÉTICA Y BIOTECNOLOGÍA DE CAMAGÜEY (CIGB). Separación de proteínas por electrofóresis en geles de poliacrilamida en presencia de SDS. PPO 4.09.004.91. Camagüey (Cuba): Centro de Ingeniería Genética y Biotecnología, 1991.

[14] CUBA. CENTRO DE INGENIERÍA GENÉTICA Y BIOTECNOLOGÍA DE CAMAGÜEY (CIGB). Tinción de proteínas en geles de policarilamida usando azul de Coomasie. PPO 4.09.005.91. Camagüey (Cuba): Centro de Ingeniería Genética y Biotecnología, 1991.

[15] CUBA. CENTRO DE INGENIERÍA GENÉTICA Y BIOTECNOLOGÍA DE CAMAGÜEY (CIGB). Determinación de pH. PPO 4.09.068.92. Camagüey (Cuba): Centro de Ingeniería Genética y Biotecnología, 1992.

[16] CUBA. CENTRO DE INGENIERÍA GENÉTICA Y BIOTECNOLOGÍA DE CAMAGÜEY (CIGB). Determinación de la concentración de timerosal. PPO 2.31.226.11. Camagüey (Cuba): Centro de Ingeniería Genética y Biotecnología, 2011.

[17] CANALES, M., ENRIQUEZ, A., RAMOS, E., CABRERA, D., DANDIE, H., SOTO, A., FALCÓN, V., RODRIGUEZ, M. and FUENTE, J. Large-scale production in Pichia pastoris of the recombinant vaccine Gavac $^{\circledR}$ against cattle tick. Vaccine, 15(4), 1997, p. 414-422. 